\title{
CÁLCULO DE TASA DE CRECIMIENTO DE TRÁNSITO PARA PROYECCIONES DE TRÁFICO PROMEDIO DIARIO ANUAL EN NICARAGUA
}

\section{CALCULATION OF TRAFFIC GROWTH RATE FOR AVERAGE DAILY ANNUAL TRAFFIC PROJECTIONS IN NICARAGUA}

Sergio Junior Navarro-Hudie/' Fernando José Bustamante Arteaga ${ }^{2}$

(Recibido/received: 20-marzo-2021; aceptado/accepted: 27-mayo-2021)

RESUMEN: El tránsito vehicular proyectado para el periodo de diseño de pavimentos es una de las más importantes variables que determinan los espesores requeridos para su diseño. Debido al comportamiento de los flujos de tránsito vehicular en las carreteras de Nicaragua, cuya referencia está indicada en los anuarios estadísticos publicados por el Ministerio de Transporte e Infraestructura, es necesario contar con un referente de la metodología para la determinación adecuada de las tasas de crecimiento a ser empleadas para calcular los Ejes de Cargas Equivalentes Simples (ESAL: Equivalent Single Axle Load) acorde al periodo de diseño por tipo de superficie seleccionada. En este artículo, basado en un estudio de caso, se desarrolla la metodología y criterios generales para la selección de un valor justificado por la dependencia de estaciones, correlaciones establecida y datos históricos publicados.

PALABRAS CLAVE: Tasa de crecimiento, estudios de tránsito, Tránsito Promedio Diario Anual

ABSTRACT: The vehicular traffic projected for the pavement design period is one of the most important variables that determine the thicknesses required for its design. Due to the behavior of vehicular traffic flows on Nicaraguan highways, the reference of which is indicated in the statistical yearbooks published by the Ministry of Transportation and Infrastructure, it is necessary to have a reference methodology for the proper determination of growth rates to be used to calculate the Simple Equivalent Single Axle Loads according to the design period by type of surface selected. In this article, based on a case study, the methodology and general criteria for the selection of a value justified by the dependence of stations, established correlations and published historical data are developed.

KEYWORDS: Growth rate, traffic studies, Average Daily Annual Traffic

1 Universidad Nacional de Ingeniería Estelí, Nicaragua. Sergio.Navarro@norte.uni.edu.ni, Orcid: https://orcid.org/0000-0002-7001-5860.

2 Universidad Nacional de Ingeniería Managua, Nicaragua. jose.bustamante@ftc.uni.edu.ni 


\section{INTRODUCCIÓN}

Como refieren Ashhad, Cabrera y Roa (2021), Cal y Mayor (2018) y Sun (2016) el Tráfico Promedio Diario Anual representa el total de vehículos que pasaron el punto específico en un año dividido por 365 , sin embargo, la proyección de los volúmenes, en particular para nuevas carreteras incluirá el tránsito normal, tránsito generado y tránsito atraído, siendo un proceso complejo el determinar tasas de crecimiento vehicular considerando la amplia variedad de vehículos y volúmenes por los distintos corredores del país.

Desde el 2005 "la red vial nacional ha sido dividida en tramos que representan secciones de carreteras donde el volumen de tráfico y carga se puede asumir uniforme sobre toda la longitud de sección de carrera", así mismo asume al tráfico normal como aquel que existirá se mejore o no el tramo, el tráfico desviado o atraído como aquel que desvía y atrae por las mejoras en carretera así como el generado que representa un volumen adicional por las mejoras y cambios en estructura de transporte (MTI, 2007).

Para Nicaragua por los altos costos incurridos no se realizan conteos clasificados de los volúmenes de tránsito durante todo el año sino divididos en tres cuatrimestres (MTI, 2019, p.9). Se considerará que el Tránsito Promedio Diario Anual será "el volumen vehicular que representa el promedio de todos los volúmenes diarios en un año" (SCT, 2016, p.11).

En Nicaragua acorde a Anuario de Aforo Tráfico 2018 publicado por el Ministerio de Transporte e Infraestructura (MTI, 2019), a través de la Oficina de diagnóstico, evaluación de pavimentos y puentes, desde el año 2010 en Nicaragua se aplican vectores de correspondencia para identificar las características, volúmenes de tráfico de las estaciones, perfil de variación en el flujo vehicular y el grado de relación entre las diferentes estaciones de la red vial.

\section{METODOLOGÍA}

Acorde a Hernández, Fernández y Baptista (2014) esta investigación es de tipo descriptiva con un enfoque cuantitativa. Para la descripción de las características y fuentes de información estadística de conteos clasificados se realizó una revisión documental, así como otros recursos encontrados en la internet de los últimos cinco años. Para el análisis y proyección de datos de tráfico clasificado así como el cálculo de tasas de crecimiento se hizo uso de la metodología propuestas en anuarios de tráfico del Ministerio de Transporte e Infraestructura de Nicaragua, aplicando de cálculo de tasas de crecimiento con valores extremos así como media geométrica para los valores positivos propuestos por MTI (2019), Barraza Ortiz, Domínguez Solano y Herrera Acosta (2019), SCT (2016) aplicados al tramo de carretera ubicado en San Sebastián de Yalí - El Tigre. 


\section{RESULTADOS Y DISCUSIÓN}

Los Anuarios de aforo de tráfico publicados por el Ministerio de Transporte son un referente del comportamiento de los volúmenes de tráfico en las carreteras del país, para las estaciones principales. A fin de homologar la nomenclatura de las estaciones de conteos clasificados y periodos de conteo manuales 0 aforos de tráfico se han clasificado estas en las categorías indicadas en la tabla 1.

Tabla 1. Estaciones de conteos clasificados en Nicaragua.

\begin{tabular}{|c|c|c|}
\hline $\begin{array}{l}\text { Estaciones de Conteos de } \\
\text { Mayor Cobertura (EMC) }\end{array}$ & $\begin{array}{l}\text { Estaciones de Conteos de } \\
\text { Corta Duración (ECD) }\end{array}$ & $\begin{array}{l}\text { Estación de Conteo } \\
\text { Sumaria (ECS) }\end{array}$ \\
\hline $\begin{array}{l}\text { Conteos clasificados durante } 3 \\
\text { tiempos por } 7 \text { días } \\
\text { consecutivos cada uno las } 24 \\
\text { horas, obteniendo el } \\
\text { Promedio Diario Anual (TPDA) } \\
\text { con el promedio de los } \\
\text { períodos. }\end{array}$ & $\begin{array}{l}\text { Conteos clasificados por } 12 \\
\text { horas continuas (6:00 a.m. - } \\
\text { 6:00 p.m.) por tres días } \\
\text { consecutivos (martes, } \\
\text { Miércoles y Jueves) } \\
\text { aplicados en flujos con } \\
\text { TPDA reportados mayores } \\
\text { de } 300 \text {. }\end{array}$ & $\begin{array}{l}\text { Conteos clasificados por } 12 \\
\text { horas continuas (6:00 a.m. - } \\
\text { 6:00 p.m.) por tres días } \\
\text { consecutivos (martes, } \\
\text { Miércoles y Jueves) aplicados } \\
\text { en flujos con TPDA } \\
\text { reportados menores de } 300 \text {. }\end{array}$ \\
\hline
\end{tabular}

Fuente: Anuario de Aforo de Tráfico Año 2018, MTI, 2019, p.9

Los vectores de correspondencia o analogía son empleados para establecer la relaciones 0 dependencias que puedan existir entre estaciones de conteos clasificados para estaciones principales y secundarias basadas en los aspectos de regiones geográficas, volumen total del tráfico, porcentaje de Vehículos Pesados en el Volumen Total Diario y la razón entre el total de Vehículos pesados de carga y vehículos de larga distancia, así como el total de vehículos pesados (MTI, 2019, p.5-7).

Los datos estadísticos de Policía Nacional (Nicaragua) indican que al 2018 la composición de toda la flota vehicular era de $88.72 \%$ correspondiente a vehículos livianos, $2.78 \%$ de pasajeros, $7.97 \%$ pesados y el $0.53 \%$ a otros. Siendo para las once principales el $77 \%$ de vehículos livianos y el resto de carga (MTI, 2019, pp. 16-19).

Lo ideal es poder realizar los conteos clasificados manuales durante toda la semana y las 24 horas, sin embardo considerando factores de costos, seguridad y disposición de recursos humanos y técnicos, es una práctica común el poder expandir datos a partir de conteos de corta duración de al menos 12 horas y al menos tres días de la semana.

La Oficina de Diagnóstico y Evaluación de Pavimentos y Puentes, a través de la División de Administración Vial refiere a que los factores para el cálculo del TPDA en las estaciones de Corta 
Duración (ECD) y estaciones Conteo Sumaria (ECS), dependiendo de los días que realice los aforos se empleen los siguientes:

Factor Día (conocido también como factor nocturno): Corresponde expandir el tráfico diurno de 12 horas a tráfico diario de 24 horas. Se obtiene mediante los resultados correspondientes de las estaciones de mayor cobertura de 24 horas siendo la fórmula:

$$
\text { Factor Día }=1+\frac{\text { Tráfico Nocturno }}{\text { Tráfico Diurno }} \text { Ecuación 1. Fuente (MTI, 2019) }
$$

El valor de factor día a adoptar por defecto en las estaciones de Mayor Cobertura 1.0.

Factor Semana: El factor para expandir el resultado obtenido para un período corto de tres días de la semana (martes a jueves) a los promedios semanales 7 días (lunes a domingo), siendo la fórmula:

Factor semana $=\left(\frac{T l a b * 5+T f s * 2}{7 * T l a b}\right)$ Ecuación 2. Fuente (MTI, 2019)

Donde:

Tlab: Corresponde al tráfico levantado durante la semana (lunes a viernes).

Tfs: Corresponden al tráfico levantado durante el fin de semana (sábado a Domingo).

Los valores de factor semana en las Estaciones de Mayor Cobertura por defecto será 1.0.

Factor Fin de Semana: Es el Factor para expandir un conteo realizado durante el fin de semana a los 7 días de la semana, siendo la fórmula para calcular:

Factor Laboral $=\left(\frac{T l a b * 5+T f s * 2}{7 * T f s}\right)$ Ecuación 3. Fuente (MTI, 2019)

Factor expansión (conocido también como factor estacional o factor de ajuste) : Es el factor para expandir el tráfico diario semanal promedio diario anual (TPDA) por tipo de vehículo, el que se obtiene de los conteos realizados en las estaciones de mayor cobertura.

Para realizar los conteos clasificados se ha estandarizado la clasificación de vehículos, pudiendo incluir otros aspectos acorde alcances del estudio, así como los pesos por eje admitidos, siendo los indicados tabla 2. 
Tabla 2. Tipología de Vehículos usada para conteos clasificados.

\begin{tabular}{|c|c|c|}
\hline Clasificación & Tipos de vehículos & Descripción de la tipología vehicular \\
\hline \multirow[t]{7}{*}{$\begin{array}{l}\text { Vehículos de } \\
\text { Pasajeros }\end{array}$} & Motocicletas & $\begin{array}{l}\text { Incluye todos los tipos de Motocicletas tales } \\
\text { como: Mini motos, Cuadraciclos, Moto Taxis, etc. } \\
\text { Este último fue modificado para que pudiera ser } \\
\text { adaptado para el traslado de personas, se } \\
\text { encuentran en zonas departamentales y zonas } \\
\text { urbanas. Moviliza a } 3 \text { personas incluyendo al } \\
\text { conductor. }\end{array}$ \\
\hline & Automoviles & $\begin{array}{l}\text { Se consideran todos los tipos de automóviles de } \\
\text { cuatro y dos puertas, entre los que podemos } \\
\text { mencionar, vehículos cope y station wagon. }\end{array}$ \\
\hline & Jeep & $\begin{array}{l}\text { Se consideran todos los tipos de vehículos } \\
\text { conocidos como } 4 \times 4 \text {. En diferentes tipos de } \\
\text { marcas, tales como Toyota, Land Rover, Jeep, } \\
\text { etc. }\end{array}$ \\
\hline & Camioneta & $\begin{array}{l}\text { Son todos aquellos tipos de vehículos con tintas } \\
\text { en la parte trasera, incluyendo las que transportan } \\
\text { pasajeros y aquellas que por su diseño están } \\
\text { destinados a trabajos de carga. }\end{array}$ \\
\hline & Microbus & $\begin{array}{l}\text { Se consideran todos aquellos microbuses, que su } \\
\text { capacidad es igual o menos a } 14 \text { pasajeros } \\
\text { sentados. }\end{array}$ \\
\hline & Minibus & $\begin{array}{l}\text { Son aquellos con una capacidad de } 15 \text { a } 30 \\
\text { pasajeros sentados. }\end{array}$ \\
\hline & Bus & $\begin{array}{l}\text { Se consideran todos los tipos de buses, para el } \\
\text { transporte de pasajeros con una capacidad de } \\
\text { más de } 30 \text { pasajeros sentados. }\end{array}$ \\
\hline \multirow[t]{2}{*}{$\begin{array}{l}\text { Vehículos de } \\
\text { Carga }\end{array}$} & Liviano de Carga & $\begin{array}{l}\text { Se consideran todos aquellos vehículos, cuyo } \\
\text { peso máximo es de } 4 \text { toneladas o menores a } \\
\text { ellas. }\end{array}$ \\
\hline & Camión de carga C2 - C3 & $\begin{array}{l}\text { Son aquellos camiones tipos C2 ( } 2 \text { ejes) y C3 ( } 3 \\
\text { ejes), con un peso mayor de } 5 \text { toneladas. } \\
\text { También se incluyen las furgonetas de carga } \\
\text { liviana. }\end{array}$ \\
\hline
\end{tabular}




\begin{tabular}{|c|c|c|}
\hline Clasificación & Tipos de vehículos & Descripción de la tipología vehicular \\
\hline & $\begin{array}{l}\text { Camión de Carga Pesada Tx- } \\
\text { Sx<=4 }\end{array}$ & $\begin{array}{l}\text { Camiones de carga pesada, son vehículos } \\
\text { diseñados para el transporte de mercancía liviana } \\
\text { y pesada. }\end{array}$ \\
\hline & Tx-Sx>=5 & $\begin{array}{l}\text { Este tipo de camiones son considerados } \\
\text { combinaciones Tractor Camión y semi- } \\
\text { Remolque, que sea mayor o igual que } 5 \text { ejes. }\end{array}$ \\
\hline & Cx-Rx<=4 & $\begin{array}{l}\text { Camión combinado, son combinaciones camión } \\
\text { remolque que sea menor o igual a } 4 \text { ejes. }\end{array}$ \\
\hline & $C x-R x>=5$ & $\begin{array}{l}\text { Son combinaciones iguales que las anteriores, } \\
\text { pero mayores o iguales cantidades a } 5 \text { ejes. }\end{array}$ \\
\hline \multirow[t]{2}{*}{ Equipo Pesado } & Vehículos Agrícolas & $\begin{array}{l}\text { Son vehículos provistos con llantas especiales de } \\
\text { hule, de gran tamaño. Muchos de estos vehículos } \\
\text { poseen arados u otros tipos de equipos, con los } \\
\text { cuales realizan actividades agrícolas. }\end{array}$ \\
\hline & Vehículos de Construcción & $\begin{array}{l}\text { Generalmente estos tipos de vehículos se utilizan } \\
\text { en la construcción de obras civiles. Pueden ser de } \\
\text { diferentes tipos: Motoniveladoras, } \\
\text { Retroexcavadora, Pavimentadora de Asfalto, } \\
\text { Tractor de Cadenas, etc. }\end{array}$ \\
\hline Otros & Remolques y/o trailers & $\begin{array}{l}\text { Se incluyen remolques o trailes pequeños } \\
\text { halados por cualquier clase de vehículo } \\
\text { automotor, también se incluyen los halados por } \\
\text { tracción animal (semovientes). }\end{array}$ \\
\hline
\end{tabular}

Fuente: $\operatorname{MTI}(2019$, p. 34)

De igual manera en correspondencia con el Reglamento de Ley General de Transporte Terrestre en los artículos 109 y 114, de las Normas Jurídicas de Nicaragua (2005) se establecen diagrama de cargas permisibles mostrado en figura 1. 

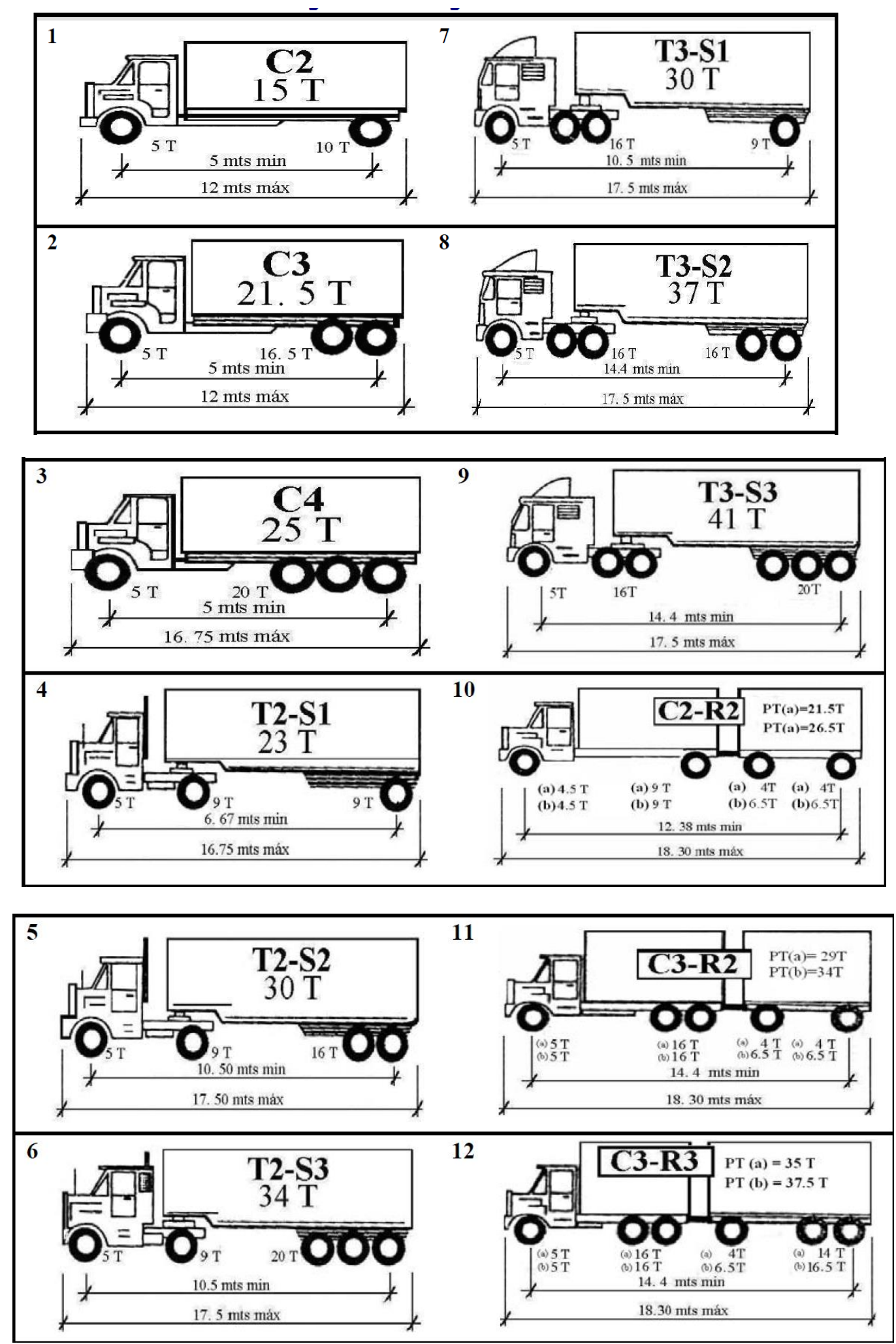

Figura 1. Diagrama de pesos permisibles en Nicaragua vehículos carga

El Higo Revista Científica / Volumen 11. No. 01, pp. 43-68/ junio 2021 
Nota: El Pesos máximo permisible será el menor entre el especificado por el fabricante y el contenido en esta columna.

(a): Eje sencillo, llanta sencilla.

(b): Eje sencillo, llanta doble

* Según vehículo cargado o descargado excederá en: Ancho: 2.60 m, Alto 4.15 m, Largo. 2 ejes $12 \mathrm{~m}, 3$ ejes $12 \mathrm{~m}$, semirremolque $17.50 \mathrm{~m}$, otras combinaciones desde $18.30 \mathrm{~m}$ hasta $23 \mathrm{~m}$ máximo. Para los ejes dobles (tandem) y triple la separación de centros comprendidos entre las ruedas es entre 1.0 y $1.30 \mathrm{~m}$.

Acorde a la División de Administración Vial de Oficina de Diagnóstico y Evaluación de Pavimentos y puentes:

Para efectos de diseño de estructuras de pavimento de acuerdo a resultados de estudios de tráfico, laboratorios de suelos, muestreos, conteo vehicular y cálculos relacionados, se han identificado que los daños que causan los vehículos livianos a la estructura del pavimento en carreteras nuevas, producto de su desplazamiento sobre la vía, es mínimo a razón de 1 en 50000 respecto a vehículos pesados, por tal motivo no figura en el diagrama de cargas permisibles de Nicaragua la información referida al respecto. Para estimaciones de cargas, seguimiento estadístico, control de tráfico y diseño de vehículos se pueden considerar los siguientes pesos por ejes, el departamento de pesos y dimensiones de la dirección vialidad del Ministerio de Transporte e Infraestructura propone la tabla de cargas de tabla 3.

Tabla 3. Diagrama de pesos permisibles en Nicaragua vehículos livianos y pasajeros

\begin{tabular}{|c|c|c|}
\hline Tipo de Vehículo & Peso por eje en TON & Peso por eje en Lb \\
\hline AUTOMOVIL & $1 / 1$ & $2200 / 2200$ \\
\hline JEEP & $1 / 1$ & $2200 / 2200$ \\
\hline CAMIONETA & $1 / 2$ & $4400 / 8800$ \\
\hline MC-15 & $2 / 4$ & $8800 / 17600$ \\
\hline MC-12-30 & $4 / 8$ & $8800 / 17600$ \\
\hline C2-LIV & $4 / 8$ & $11000 / 22000$ \\
\hline BUS $=$ C2 & $5 / 10$ & $2200 / 4400$ \\
\hline
\end{tabular}

El Higo Revista Científica / Volumen 11. No. 01, pp. 43-68/ junio 2021 
La División de Administración Vial de Oficina de Diagnóstico y Evaluación de Pavimentos y Puentes (MTI, 2019, pp. 16-17) refiere que el método más común para la Proyección de Tráfico es la ecuación siguiente:

$$
T C=\left[\left(\frac{T P D A_{i}}{T P D A_{0}}\right)^{1 / n}\right]-1 \text { Ecuación 4. Fuente (MTI, 2019) }
$$

Donde:

$\mathrm{TC}=$ Tasa de Crecimiento

$\mathrm{TPDA}_{\mathrm{i}}=$ Tráfico Promedio Diario Actual.

$\mathrm{TPDA}_{0}=$ Tráfico Promedio Diario Actual del Año Base.

$\mathrm{n}=\mathrm{La}$ diferencia de años

Una vez obtenida una serie de tasas de un año con otro se procede a aplicar la media geométrica aplicando la siguiente ecuación.

$T f=\left[\left(T C_{1} * T C_{2} * \ldots * T C_{n}\right)^{1 / n}\right]$ Ecuación 5. Fuente (MTI, 2019)

Donde:

Tf: Tasa de Crecimiento Final

$T c_{n}$ : Diversas tasas de crecimiento obtenidas de la serie histórica, tomando los datos evaluadas en periodos de crecimiento o valores positivos.

$\mathrm{n}$ : cantidad de tasas de crecimiento

La media geométrica es una medida de tendencia central que puede utilizarse para mostrar los cambios porcentuales en una serie de números positivos (Barraza Ortiz, Domínguez Solano y Herrera Acosta, 2019).

Como un referente de los valores de tasas de crecimiento general para las estaciones, durante el periodo 2010 -2018 se evaluó que las tasas de crecimiento ponderadas están en rangos desde $3.36 \%$ cuando iniciaron las nuevas estaciones hasta un $6 \%$ (MTI, 2019, p.18).

Para este artículo se tomó como ejemplo de aplicación práctica el análisis del tramo de carretera San Sebastián de Yalí - El Tigre se encuentra localizado en el departamento de Jinotega, en el corredor San Sebastián de Yalí - La Rica correspondiente a la clasificación NIC-35D con una estación de corta duración código 3503 cuya dependencia es la Estación de Mayor cobertura 1802 (San Marcos Masatepe), mostrado en figura 2 y tabla 4. 


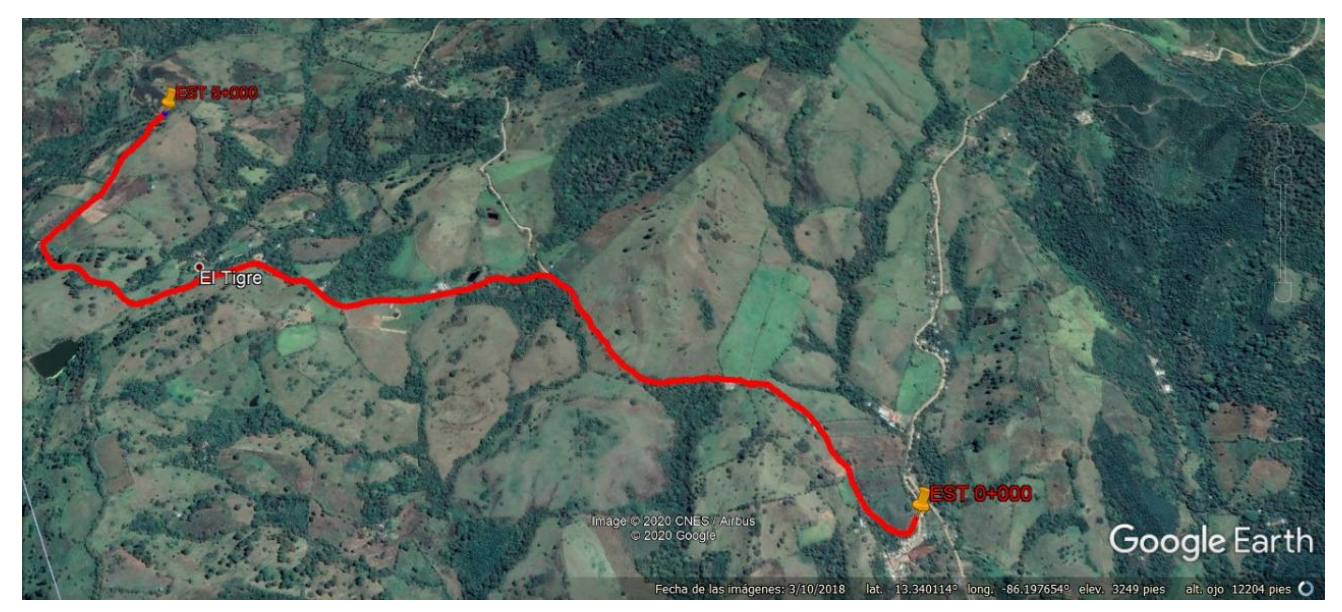

Figura 2. Localización de tramo carretero referencia. Fuente: Google Earth

Tabla 4. Dependencia de estaciones Fuente: Anuario de Aforo de Tráfico año 2018, (MTI, 2018) (MTI, 2019,9. 68).

\begin{tabular}{|c|c|c|c|c|c|}
\hline $\begin{array}{l}\text { Estación de } \\
\text { mayor } \\
\text { cobertura }\end{array}$ & NIC & $\begin{array}{c}\text { No. } \\
\text { Estación }\end{array}$ & Tipo & Pkm & Nombre del tramo \\
\hline \multirow{10}{*}{$\begin{array}{l}1802 \text { San } \\
\text { Marcos - } \\
\text { Masatepe }\end{array}$} & NIC-35B & 3507 & ECS & 164.2 & $\begin{array}{l}\text { Emp. Chilamatillo }- \text { Escuela } \\
\text { Miraflores }\end{array}$ \\
\hline & NIC-35B & 3508 & ECS & 180.0 & $\begin{array}{l}\text { Escuela Miraflores- San Sebastián } \\
\text { de Yalí (Empalme Quiatas) }\end{array}$ \\
\hline & NIC-35C & 2605 & ECS & 166.0 & El Jicaral - Santa Rosa del Peñon \\
\hline & NIC-35D & 3503 & ECD & 206.4 & San Sebastián de Yalí - La Rica \\
\hline & NIC-36 & 1214 & ECD & 115.0 & Emp. Posoltega - Posoltega \\
\hline & NIC-37A & 714 & ECS & 145.0 & $\begin{array}{l}\text { Las Lajitas-Cuapa-Comarca El } \\
\text { Zancudo }\end{array}$ \\
\hline & NIC-37B & 3701 & ECS & 140.0 & Juigalpa - Puerto Diaz \\
\hline & NIC-38 & 3801 & ECS & 164.0 & Dos Montes - El Sauce \\
\hline & NIC-38 & 2611 & ECS & 185.6 & El Sauce - Río Grande \\
\hline & NIC-38 & 3802 & ECS & 200.0 & Río Grande - Achuapa \\
\hline
\end{tabular}

Los pasos a seguir, sugeridos para la determinación de tasa de crecimiento se van desarrollando en orden secuencial, mismos que se desarrollan como referente. Primer paso: Se realizó un conteo manual clasificado de 12 horas durante una semana completa, realizado el cálculo de TPDS con el promedio de los siete días aforados, mostrados en la tabla 5. 
Tabla 5. Conteo Manual Clasificado.

\begin{tabular}{|c|c|c|c|c|c|c|c|c|c|c|c|c|}
\hline \multirow{2}{*}{\multicolumn{2}{|c|}{ Hora }} & \multicolumn{4}{|c|}{ Vehículos livianos } & \multicolumn{3}{|c|}{ Vehículos de pasajeros } & \multicolumn{3}{|c|}{ Vehículos de carga } & \multirow{3}{*}{ TOTAL } \\
\hline & & \multirow{2}{*}{ Motocicletas } & \multirow{2}{*}{ Automóvil } & \multicolumn{2}{|c|}{ 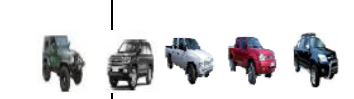 } & \multirow{2}{*}{$\begin{array}{l}\text { An } \\
\text { Microbus }\end{array}$} & \multirow{2}{*}{$\begin{array}{l}\text { MinuBus } \\
\text { M }\end{array}$} & \multirow{2}{*}{$\begin{array}{l}2 \\
\text { Bus }\end{array}$} & \multirow{2}{*}{$\begin{array}{c}\text { Liviano } \\
\text { de } \\
\text { Carga } \\
\end{array}$} & \multirow{2}{*}{$\begin{array}{l}\text { 달 } \\
\text { C2 }\end{array}$} & \multirow[b]{2}{*}{ C3 } & \\
\hline Inicio & Fin & & & Jeep & Camionetas & & & & & & & \\
\hline 08:00 & 09:00 & 67 & 2 & 0 & 28 & 0 & 0 & 3 & 17 & 0 & 1 & 120 \\
\hline 09:00 & $10: 00$ & 60 & 6 & 0 & 20 & 0 & 0 & 3 & 10 & 15 & 1 & 120 \\
\hline $10: 00$ & $11: 00$ & 59 & 6 & 1 & 24 & 1 & 0 & 3 & 8 & 4 & 2 & 110 \\
\hline $11: 00$ & $12: 00$ & 45 & 10 & 0 & 19 & 0 & 0 & 3 & 10 & 4 & 3 & 104 \\
\hline $14: 00$ & $15: 00$ & 84 & 16 & 1 & 18 & 1 & 0 & 8 & 8 & 11 & 0 & 149 \\
\hline $15: 00$ & $16: 00$ & 63 & 32 & 3 & 24 & 1 & 0 & 8 & 12 & 26 & 2 & 176 \\
\hline $16: 00$ & $17: 00$ & 57 & 28 & 2 & 17 & 1 & 0 & 11 & 12 & 41 & 1 & 177 \\
\hline $17: 00$ & $18: 00$ & 72 & 42 & 0 & 50 & 0 & 0 & 6 & 39 & 41 & 5 & 257 \\
\hline \multicolumn{2}{|c|}{ TOTAL } & 756 & 192 & 9 & 316 & 4 & 0 & 70 & 165 & 231 & 21 & 1807 \\
\hline
\end{tabular}

Fuente: Datos de campo por estudiantes Oliver Rivera, Pedro Hurtado y Luis Hernández 
Paso 2. Identificar estaciones de referencias para usar factores de expansión.

Es necesario que se identifique en los anuarios la estación de conteo más cercana que permita a través de aforos previos establecer los factores de ajuste apropiados, en particular los factores días, considerando que en las estaciones principales el factor día y factor semana es de 1, lo cual refiere a que el conteo fue realizado completo por semana.

Basado en los planteado por Anuario de Aforo de Tráfico (MTI, 2019) el TPDA es calculado por la ecuación:

TPDA $=\operatorname{TP}(\mathrm{D}){ }^{*}$ Factor Día *Factor Ajuste $\quad$ Ecuación 6. Fuente (MTI, 2019)

Donde TP(D) es el Tránsito promedio diurno, mismo que será equivalente al Tráfico Promedio Diario Semanal cuando se afora la semana completa durante las 12 horas.

En el caso de contar solo 3 días podrán determinarse el TPDA por la expresión: TPDA $=T P(D)$ * Factor Día * Factor Semana * Factor Ajuste

Estos factores de ajuste son también un referente para otros diseños como cargas en puentes siendo referidos a "coeficientes de fluctuación diaria, semanal y estacional que posee el Ministerio de Transporte e Infraestructura" (JICA, 2014, p. 2-33).

Si no se encuentra estaciones de referencia, será necesario hacer uso de estaciones de dependencia. Para este ejemplo, dado que el conteo fue realizado en febrero 2021, se podría emplear los factores correspondientes al primer cuatrimestre del año, tabla 6. 
Tabla 6. Estación de conteo de mayor cobertura realizada por el MTI. Fuente: Anuario de Aforo de Tráfico año 2018, (MTI, 2018) (MTI, 2019, p. 346).

Conteo 1

\begin{tabular}{|c|c|c|c|c|c|c|c|c|c|c|c|c|c|c|c|c|c|c|}
\hline \multirow{4}{*}{$\begin{array}{l}\text { Camino } \\
: \quad \text { NIC- } \\
18 \mathrm{~A} \\
\text { Grupos }\end{array}$} & \multicolumn{18}{|c|}{ Estación: 1802 Tramo: San Marcos - Masatepe Periodo: S Días: 7 Horas: Mes: Mar. Año: 2018 KM: 46.000} \\
\hline & \multirow[t]{2}{*}{ Motos } & \multicolumn{6}{|c|}{ Vehículos de Pasajeros } & \multicolumn{7}{|c|}{ Vehículos de carga } & \multicolumn{2}{|c|}{$\begin{array}{l}\text { Equipo } \\
\text { pesado }\end{array}$} & \multirow[t]{2}{*}{ Otros } & \multirow[t]{3}{*}{ Total } \\
\hline & & Autos & Jeep & Cam. & $\begin{array}{c}\text { McBus } \\
<15\end{array}$ & $\begin{array}{c}\text { MnBus } \\
15-30\end{array}$ & $\begin{array}{l}\text { Bus } \\
30+\end{array}$ & $\begin{array}{l}\text { Liv. } \\
2-5 \mathrm{t} .\end{array}$ & $\begin{array}{l}\mathrm{C} 2 \\
5+\mathrm{t} .\end{array}$ & C3 & $\begin{array}{l}\text { Tx-Sx } \\
<=4 \text { e. }\end{array}$ & $\begin{array}{l}\text { Tx-Sx } \\
>=5 \text { e. }\end{array}$ & $\begin{array}{l}\text { Cx-Rx } \\
<=4 \text { e. }\end{array}$ & $\begin{array}{l}\text { Cx-Rx } \\
>=5 \text { e. }\end{array}$ & V. A & V.C & & \\
\hline & 2 & 3 & 4 & 5 & 6 & 7 & 8 & 10 & 11 & 12 & 13 & 14 & 15 & 16 & 18 & 19 & 21 & \\
\hline TP (D) & 5184 & 1691 & 364 & 1156 & 406 & 98 & 176 & 473 & 228 & 58 & & 114 & & & & & 9 & 9957 \\
\hline $\begin{array}{l}\text { Factor } \\
\text { día }\end{array}$ & 1.00 & 1.00 & 1.00 & 1.00 & 1.00 & 1.00 & 1.00 & 1.00 & 1.00 & 1.00 & 1.00 & 1.00 & 1.00 & 1.00 & 1.00 & 1.00 & 1.00 & \\
\hline $\begin{array}{l}\text { Factor } \\
\text { semana }\end{array}$ & 1.00 & 1.00 & 1.00 & 1.00 & 1.00 & 1.00 & 1.00 & 1.00 & 1.00 & 1.00 & 1.00 & 1.00 & 1.00 & 1.00 & 1.00 & 1.00 & 1.00 & \\
\hline $\begin{array}{l}\text { Factor } \\
\text { ajuste }\end{array}$ & 1.00 & 0.92 & 0.88 & 0.89 & 0.95 & 1.00 & 0.90 & 0.91 & 0.90 & 0.92 & 1.00 & 0.81 & 1.00 & 1.00 & 1.00 & 1.00 & 0.77 & \\
\hline $\begin{array}{l}\text { TPDA } \\
\text { Ene-Abr }\end{array}$ & 5167 & 1548 & 319 & 1032 & 386 & 98 & 159 & 431 & 205 & 54 & & 93 & & & & & 7 & 9,499 \\
\hline $\begin{array}{c}\% \\
\text { TPDA }\end{array}$ & 54.40 & 16.30 & 3.36 & 10.86 & 4.06 & 1.03 & 1.67 & 4.54 & 2.16 & 0.57 & & 0.98 & & & & & 0.07 & 100.00 \\
\hline \multicolumn{7}{|c|}{ \% Vehículos Livianos } & \multicolumn{8}{|c|}{ \% Vehículos pesados $9.92 \%$} & & & $0.07 \%$ & $100.00 \%$ \\
\hline
\end{tabular}


Misma que contó con tres periodos de aforo en el año, siendo los presentados en tabla 7.

Tabla 7. Factores de expansión por cuatrimestre. Fuente: Anuario de Aforo de Tráfico año 2018, (MTI, 2018) (MTI, 2019, p. 68).
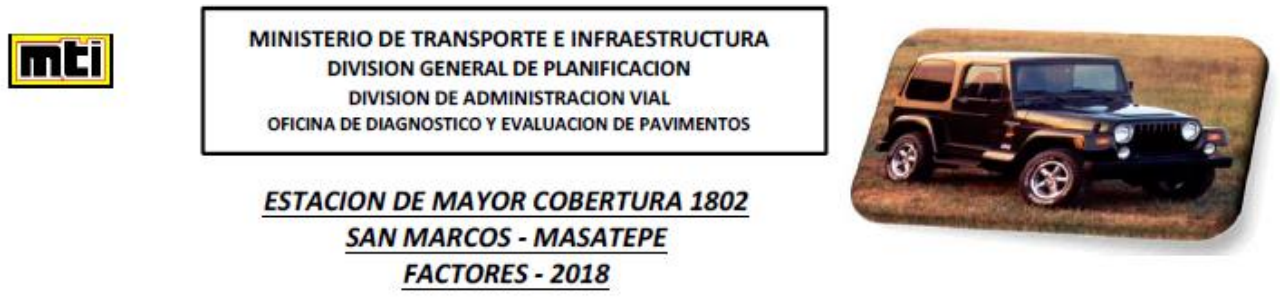

FACTORES - 2018

Factores del primer cuatrimestre del año Enero - Abril

\begin{tabular}{|c|c|c|c|c|c|c|c|c|c|c|c|c|c|c|c|c|c|}
\hline Descripción & Moto & Carro & Jeep & Camioneta & $\begin{array}{l}\text { Micro } \\
\text { Bus }\end{array}$ & $\begin{array}{l}\text { Mini } \\
\text { Bus }\end{array}$ & Bus & $\begin{array}{l}\text { Liv. } \\
2-5 \\
\text { t. }\end{array}$ & $\mathrm{C2}$ & C3 & $\begin{array}{l}\text { Tx- } \\
S x<=4\end{array}$ & $\begin{array}{l}T x- \\
S x=>5\end{array}$ & $\begin{array}{l}\text { Cx- } \\
R x=<4\end{array}$ & $\begin{array}{l}\text { Cx- } \\
R x=>5\end{array}$ & V.A & V.C & Otros \\
\hline Factor día & 1.42 & 1.47 & 1.30 & 1.33 & 1.37 & 1.31 & 1.31 & 1.21 & 1.26 & 1.18 & 1.00 & 1.56 & 1.00 & 1.00 & 1.00 & 1.00 & 1.13 \\
\hline $\begin{array}{l}\text { Factor } \\
\text { semana }\end{array}$ & 0.99 & 1.08 & 1.09 & 1.04 & 0.97 & 0.97 & 0.94 & 0.90 & 0.85 & 0.83 & 1.00 & 0.84 & 1.00 & 1.00 & 1.00 & 1.00 & 0.90 \\
\hline $\begin{array}{l}\text { Factor fin de } \\
\text { semana }\end{array}$ & 1.02 & 0.84 & 0.82 & 0.92 & 1.09 & 1.10 & 1.21 & 1.36 & 1.78 & 2.01 & 1.00 & 1.87 & 1.00 & 1.00 & 1.00 & 1.00 & 1.41 \\
\hline $\begin{array}{l}\text { Factor } \\
\text { expansión a } \\
\text { TPDA }\end{array}$ & 1.00 & 0.92 & 0.88 & 0.89 & 0.95 & 1.00 & 0.90 & 0.91 & 0.90 & 0.92 & 1.00 & 0.81 & 1.00 & 1.00 & 1.00 & 1.00 & 0.77 \\
\hline
\end{tabular}


Factores del segundo cuatrimestre del año Mayo - Agosto

\begin{tabular}{|c|c|c|c|c|c|c|c|c|c|c|c|c|c|c|c|c|c|}
\hline Descripción & Moto & Carro & Jeep & Camioneta & $\begin{array}{l}\text { Micro } \\
\text { Bus }\end{array}$ & $\begin{array}{l}\text { Mini } \\
\text { Bus }\end{array}$ & Bus & $\begin{array}{l}\text { Liv. } \\
2-5 \\
\text { t. }\end{array}$ & $\mathrm{C2}$ & C3 & $\begin{array}{c}\text { Tx- } \\
S x<=4\end{array}$ & $\begin{array}{c}T x- \\
S x=>5\end{array}$ & $\begin{array}{c}C x- \\
R x=<4\end{array}$ & $\begin{array}{c}C x- \\
R x=>5\end{array}$ & V.A & V.C & Otros \\
\hline Factor día & 1.36 & 1.32 & 1.20 & 1.24 & 1.32 & 1.24 & 1.32 & 1.16 & 1.21 & 1.14 & 1.00 & 1.19 & 1.00 & 1.00 & 1.00 & 1.00 & 1.20 \\
\hline $\begin{array}{l}\text { Factor } \\
\text { semana }\end{array}$ & 0.98 & 1.04 & 1.03 & 1.02 & 0.95 & 0.97 & 0.91 & 0.91 & 0.86 & 1.00 & 1.00 & 0.90 & 1.00 & 1.00 & 1.00 & 1.00 & 1.06 \\
\hline $\begin{array}{l}\text { Factor fin de } \\
\text { semana }\end{array}$ & 1.06 & 0.90 & 0.93 & 0.96 & 1.16 & 1.08 & 1.34 & 1.30 & 1.70 & 1.00 & 1.00 & 1.40 & 1.00 & 1.00 & 1.00 & 1.00 & 0.88 \\
\hline $\begin{array}{l}\text { Factor } \\
\text { Expansión a } \\
\text { TPDA }\end{array}$ & 1.00 & 1.20 & 1.11 & 1.15 & 1.06 & 1.01 & 1.09 & 1.08 & 1.16 & 0.94 & 1.00 & 1.33 & 1.00 & 1.00 & 1.00 & 1.00 & 1.44 \\
\hline
\end{tabular}

Factores del tercer cuatrimestre del año septiembre - Diciembre

\begin{tabular}{|c|c|c|c|c|c|c|c|c|c|c|c|c|c|c|c|c|c|}
\hline Descripción & Moto & Carro & Jeep & Camioneta & $\begin{array}{l}\text { Micro } \\
\text { Bus }\end{array}$ & $\begin{array}{l}\text { Mini } \\
\text { Bus }\end{array}$ & Bus & $\begin{array}{l}\text { Liv. } \\
2-5 \\
\text { t. }\end{array}$ & $\mathrm{C2}$ & C3 & $\begin{array}{c}\text { Tx- } \\
\text { Sx<=4 }\end{array}$ & $\begin{array}{c}\text { Tx- } \\
S x=>5\end{array}$ & $\begin{array}{c}\mathrm{CX}- \\
\mathrm{Rx}=<4\end{array}$ & $\begin{array}{c}\mathrm{Cx}- \\
\mathrm{Rx}=>5\end{array}$ & V.A & V.C & Otros \\
\hline Factor día & 1.44 & 1.41 & 1.21 & 1.28 & 1.34 & 1.29 & 1.32 & 1.23 & 1.25 & 1.07 & 1.00 & 1.22 & 1.00 & 1.00 & 1.00 & 1.00 & 1.00 \\
\hline $\begin{array}{l}\text { Factor } \\
\text { semana }\end{array}$ & 0.99 & 1.12 & 1.11 & 1.03 & 0.97 & 0.99 & 0.98 & 0.92 & 0.86 & 0.89 & 1.00 & 0.85 & 1.00 & 1.00 & 1.00 & 1.00 & 0.96 \\
\hline $\begin{array}{l}\text { Factor fin de } \\
\text { semana }\end{array}$ & 1.03 & 0.79 & 0.80 & 0.94 & 1.10 & 1.01 & 1.05 & 1.30 & 1.73 & 1.45 & 1.00 & 1.78 & 1.00 & 1.00 & 1.00 & 1.00 & 1.10 \\
\hline $\begin{array}{l}\text { Factor } \\
\text { Expansión a } \\
\text { TPDA }\end{array}$ & 1.00 & 0.93 & 1.04 & 0.99 & 1.00 & 0.99 & 1.03 & 1.02 & 0.98 & 1.16 & 1.00 & 0.98 & 1.00 & 1.00 & 1.00 & 1.00 & 0.99 \\
\hline
\end{tabular}


Al buscar en referencia los anuarios de aforo se encontró que los datos de la estación realizada en el mes de Julio 2015 , la cual es la estación que incluye los factores de expansión, mismos que podrán ser empleados para proyectar considerando la representación geográfica, tomando en consideración que es la estación de mayor cobertura empleada, tabla 8.

Tabla 8. Estación de conteo sumaria. Fuente: Fuente: Anuario de Aforo de Tráfico año 2015, (MTI, 2016, p. 383).

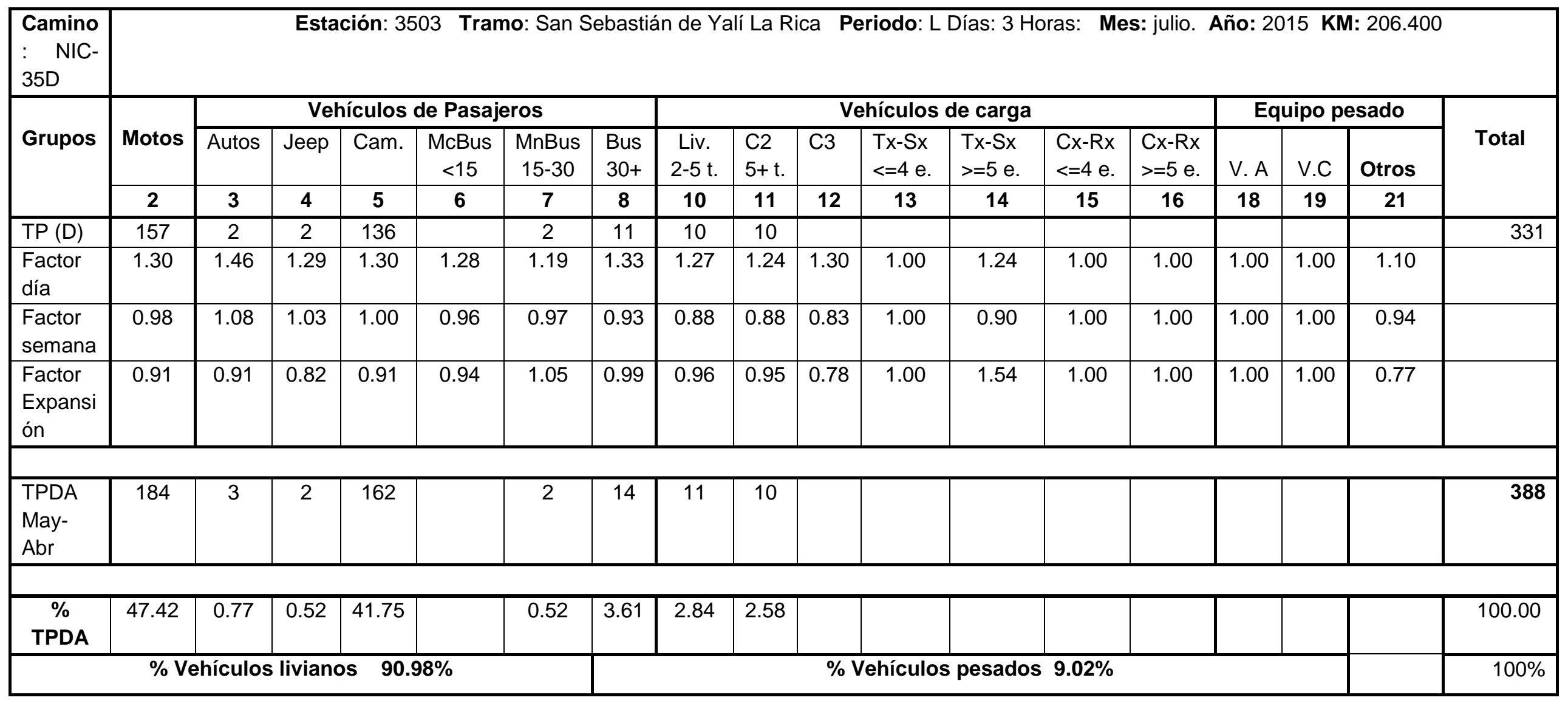


Al emplear estos factores y comparar los resultados al usar la estación de corta duración y de mayor cobertura, existe un $27 \%$ de diferencia entre ambas perspectivas de estimación en el total de tráfico y en particular $31 \%$ para los tipos $\mathrm{C} 2$, siendo significativo en las condiciones de carga impuestas, figura 3.

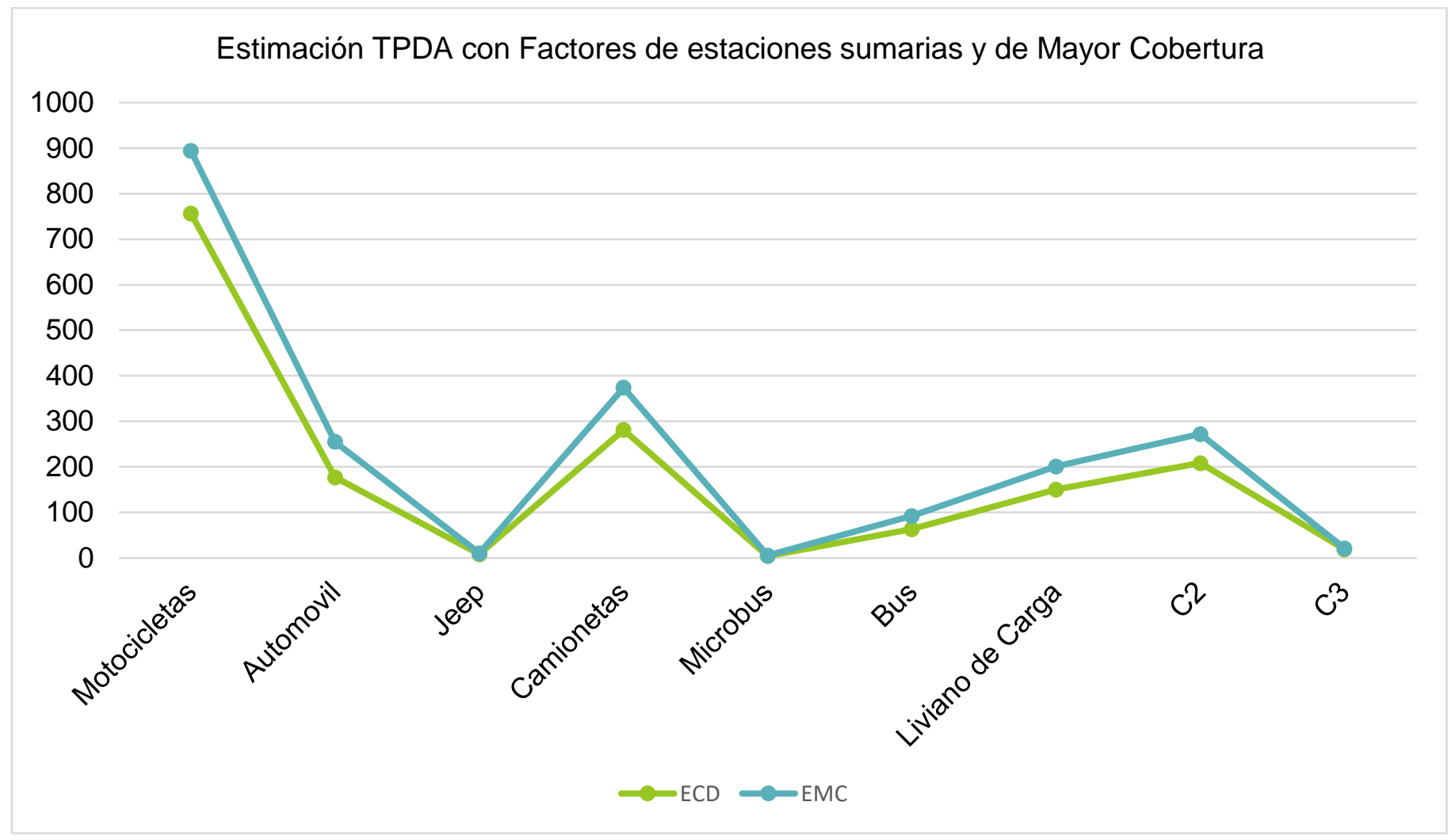

Figura 3. Comparación de estaciones de TPDA por estaciones

Paso 3. Establecer la tasa de crecimiento basado en estación de conteo seleccionada

En este paso, se deberá de analizar el comportamiento de las tasas de crecimiento, de la estación seleccionada para usar los factores de expansión (Factor día, factor semana, factor expansión). En el caso de usar en análisis estaciones de mayor cobertura, se evaluarán por medias geométricas, tal y como se desarrolla a continuación:

Basado en anuarios de aforo de tráfico 2018 (MTI, 2019,p. 176) los históricos de TPDA de estación 3503, de San Sebastián de Yalí. Este análisis se hará y los resultados podrán compararse con los indicadores referenciales del crecimiento de tasas de crecimiento generales, así como en particular, siendo para esta estación de 7.32\% 
Tabla 9. Datos históricos de estación sumaria por tipo de vehículos

\begin{tabular}{|c|c|c|c|c|c|c|c|c|c|}
\hline Año & Motos & Autos & Jeep & Cmta. & $\begin{array}{c}\text { MnBus } \\
15-30 \\
\text { S. }\end{array}$ & Bus & $\begin{array}{c}\text { Liv. } \\
2-5 \\
\text { Ton }\end{array}$ & $\begin{array}{c}\text { C2 } \\
5+ \\
\text { Ton }\end{array}$ & TPDA \\
\hline 2018 & 227 & 4 & 2 & 200 & 2 & 17 & 14 & 12 & 480 \\
\hline 2015 & 184 & 3 & 2 & 162 & 2 & 14 & 11 & 10 & 388 \\
\hline 2009 & 187 & & 10 & 103 & & 19 & 2 & 18 & 342 \\
\hline 2006 & 33 & & 6 & 69 & & 17 & 6 & 15 & 146 \\
\hline 2002 & 28 & 1 & 12 & 80 & & 15 & 16 & 15 & 168 \\
\hline 1999 & 24 & & 10 & 72 & & 22 & 6 & 9 & 143 \\
\hline
\end{tabular}

Fuente: Anuario de Aforo de Tráfico año 2018, (MTI, 2018) (MTI, 2019, p. 176)

De estos datos se evaluará la tasa de crecimiento extrayendo los años y valores de TPDA de tabla 10.

Tabla 10. Datos históricos de TPDA disponibles para estación sumaria

\begin{tabular}{|l|l|}
\hline AÑO & TPDA \\
\hline 1999 & 143 \\
\hline 2002 & 168 \\
\hline 2006 & 146 \\
\hline 2009 & 342 \\
\hline 2015 & 388 \\
\hline 2018 & 480 \\
\hline
\end{tabular}

Aplicando la ecuación se determina las tasas de crecimiento interanuales, evaluando sola las positivas. Es importante recordar que a criterio del consultor o evaluador podrá excluir ciertos años, en los cuales, debido a condiciones atípicas fuera de control como los fenómenos sociopolíticos, podrán ser excluidos de este análisis. A modo de ejemplo la tasa interanual de crecimiento entre los años 2015-2018.

$$
T C=\left[\left(\frac{480}{388}\right)^{1 /(2018-2015)}\right]-1=0.07350=7.35 \%
$$

El Higo Revista Científica / Volumen 11. No. 01, pp. 43-68/ junio 2021 
En la siguiente tabla se muestra la tasa de crecimiento por periodos.

Tabla 11. Tasa de crecimiento por periodos

\begin{tabular}{|c|c|c|c|}
\hline AÑO & TPDA & Período & TC \\
\hline 1999 & 143 & $1999-2002$ & $5.52 \%$ \\
\hline 2002 & 168 & $2002-2006$ & $-3.45 \%$ \\
\hline 2006 & 146 & $2006-2009$ & $32.81 \%$ \\
\hline 2009 & 342 & $2009-2015$ & $2.13 \%$ \\
\hline 2015 & 388 & $2015-2018$ & $7.35 \%$ \\
\hline 2018 & 480 & & \\
\hline
\end{tabular}

Basados en estos datos, se tomaron como criterios de exclusión los valores negativos, y las tasas que exceden $5 \%$ los valores máximos de referencia de estaciones de Mayor Cobertura acorde a los anuarios estadísticos. Para este caso, se excluye -3.45 y el valor de 32.81 y se dejarán los datos de referencia positivos que se calcularon. Con estos datos se procede a determinar la media geométrica la cual resultará:

$T f=\left[(5.52 * 2.13 * 7.35)^{1 / 3}\right]=4.42 \%$

Se sugiere que al menos se dispongan de 5 datos de análisis, para ello se podrá usar otros periodos de análisis considerando otro año base de referencia. Para esto se emplearán las relaciones de últimos cinco datos reflejados, así para este ejemplo se usará periodo 2006-2015, 2006-2018 cuyas tasas son:

$$
\begin{aligned}
& \mathrm{TC}=\left[\left(\frac{388}{146}\right)^{1 /(2015-2006)}\right]-1=0.11472=11.47 \% \\
& \mathrm{TC}=\left[\left(\frac{480}{146}\right)^{1 /(2018-2006)}\right]-1=0.10427=10.43 \%
\end{aligned}
$$

Por tanto, la tasa de crecimiento a ser empleada será de:

$\mathrm{Tf}=\left[(5.52 * 2.13 * 7.35 * 11.47 * 10.43)^{1 / 5}\right]=6.35 \%$, valor que corresponde a los rangos promedios de la estación de mayor cobertura. Siendo esta la tasa general de crecimiento, es decir la tasa de crecimiento para todos los vehículos.

Acorde a condiciones de tráfico y tendencias de crecimiento y reducir el efecto del crecimiento desproporcionado entre los vehículos de carga y livianos. Se pueden analizar las tasas de crecimiento promedios registradas como un referente y luego encontrar correlaciones con datos económicos de Producto Interno Bruto y crecimiento poblacional. 
En Nicaragua, el Banco Central de Nicaragua, publica anuarios que recogen "información estadística de los principales sectores económicos del país, y permite proporcionar un horizonte general del comportamiento económico" (BCN, 2021). Estas relaciones tratan de establecer relaciones, asociando el tráfico de carga al crecimiento del producto interno bruto y el de pasajeros al transporte considerando propuestas de Díaz (2020), Jordá y Lopez (2020), López y Pardo (2019), Rendón, Hernández y Del Rio, 2019) Berrones, Canos, Sánchez y Martínez (2018), OCDE (2018), Machado (2017), Rodríguez, Mejía y Zapata (2015) y Zamora y Pedraza (2013).

Por lo anterior se establecerán correlaciones entre TPDA y PIB, así como TPDA y POB, calculando las tasas anuales de crecimiento promedio para luego correlacionarlas con resultados de correlaciones generadas en Excel mostrado en tabla 12.

Tabla 12. Tasas de crecimiento de PIB y Población.

\begin{tabular}{|c|c|c|c|c|c|c|}
\hline AÑO & 143 & $\begin{array}{c}\text { Producto } \\
\text { Interno } \\
\text { Bruto } \\
\text { (PIB) } \\
26,008.91\end{array}$ & $\begin{array}{c}\begin{array}{c}\text { Tasa de } \\
\text { Crecimiento }\end{array} \\
2.60 \%\end{array}$ & $\begin{array}{c}\text { Población } \\
\text { (POB) } \\
5,016.89\end{array}$ & $\begin{array}{c}\begin{array}{c}\text { Tasa de } \\
\text { Crecimie } \\
\text { nto }\end{array} \\
1.49 \%\end{array}$ & IPC \\
\hline 2002 & 168 & $28,087.54$ & $43.42 \%$ & $5,244.69$ & $1.82 \%$ & 69.90 \\
\hline 2006 & 146 & $118,837.71$ & $1.67 \%$ & $5,637.97$ & $1.24 \%$ & 95.51 \\
\hline 2009 & 342 & $124,907.70$ & $5.28 \%$ & $5,850.50$ & $1.14 \%$ & 131.86 \\
\hline 2015 & 388 & $170,093.16$ & $1.74 \%$ & $6,262.71$ & $1.08 \%$ & 190.34 \\
\hline 2018 & 480 & 179,107 & & $6,467.70$ & & 214.76 \\
\hline & $\begin{array}{l}\text { Tasas } \\
\text { promedio }\end{array}$ & & $2.82 \%$ & & $1.36 \%$ & \\
\hline
\end{tabular}

Nota: La tasa de crecimiento de PIB del periodo

La tasa de crecimiento fue calculada con el promedio de las tasas de crecimiento de los años en estudio, figura 4. 

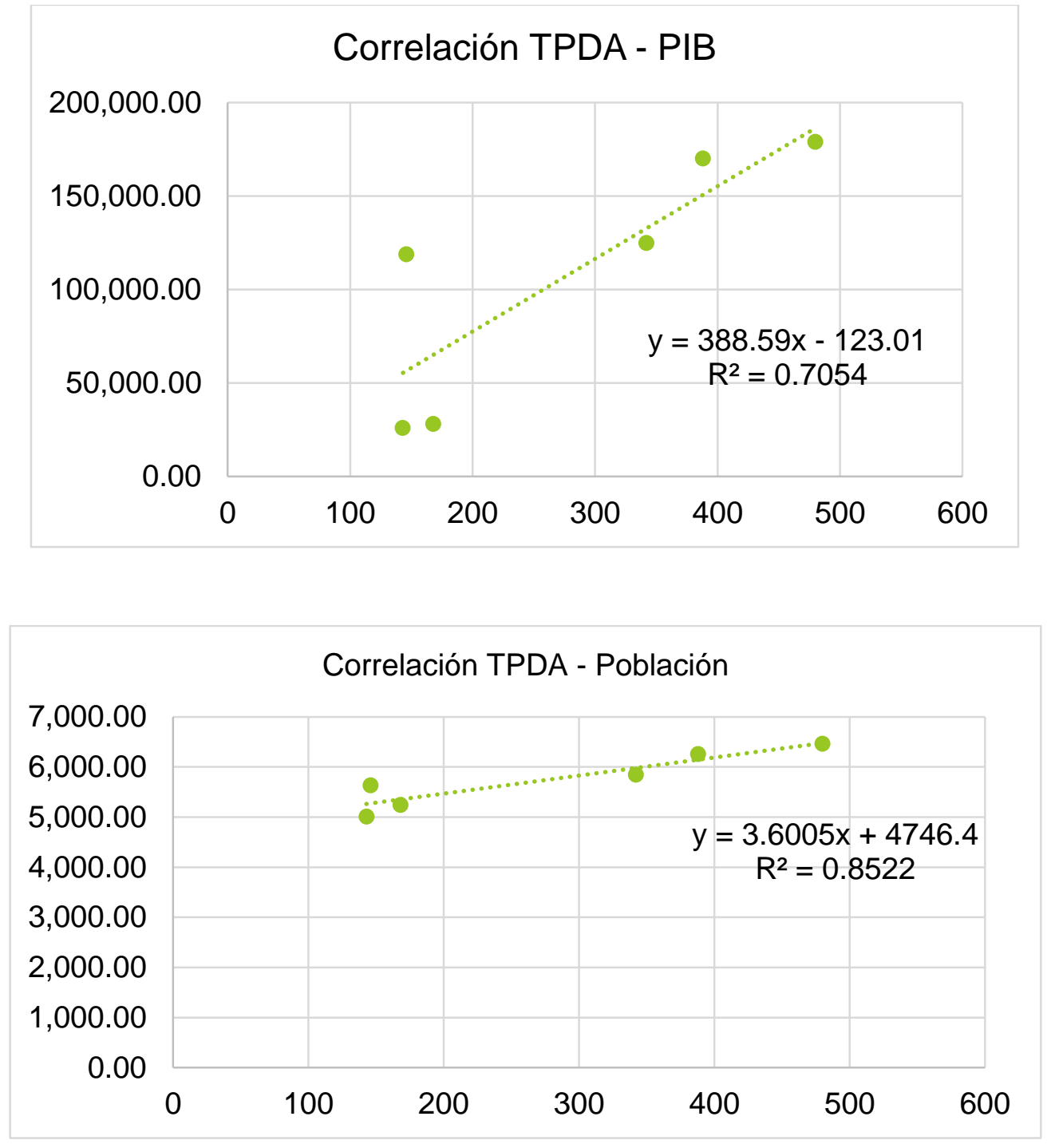

Figura 4. Correlaciones entre TPDA y datos macroeconómicos

Aunque los valores de coeficientes de ajuste derivados de las correlaciones son mayores de 0.5, se genera un modelo matemático basado en transformaciones de logaritmo natural para evaluar el valor de pendiente e intentar a partir de tasa media de crecimiento para población y PIB multiplicar por coeficiente de ajuste relación de pendiente generado. Al generar este modelo, tabla 10 y figura 5 . 
Sergio J. Navarro H. y Fernando J. Bustamante A.

Tabla 10. Logaritmos naturales de datos de TPDA, PIB y Población.

\begin{tabular}{|c|c|c|c|}
\hline AÑO & LT TPDA & LN PIB & LN POB \\
\hline 1999 & 4.96 & 10.17 & 8.52 \\
\hline 2002 & 5.12 & 10.24 & 8.56 \\
\hline 2006 & 4.98 & 11.69 & 8.64 \\
\hline 2009 & 5.83 & 11.74 & 8.67 \\
\hline 2015 & 5.96 & 12.04 & 8.74 \\
\hline 2018 & 6.17 & 12.10 & 8.77 \\
\hline
\end{tabular}

\section{Correlación Ln TPDA y Ln PIB}
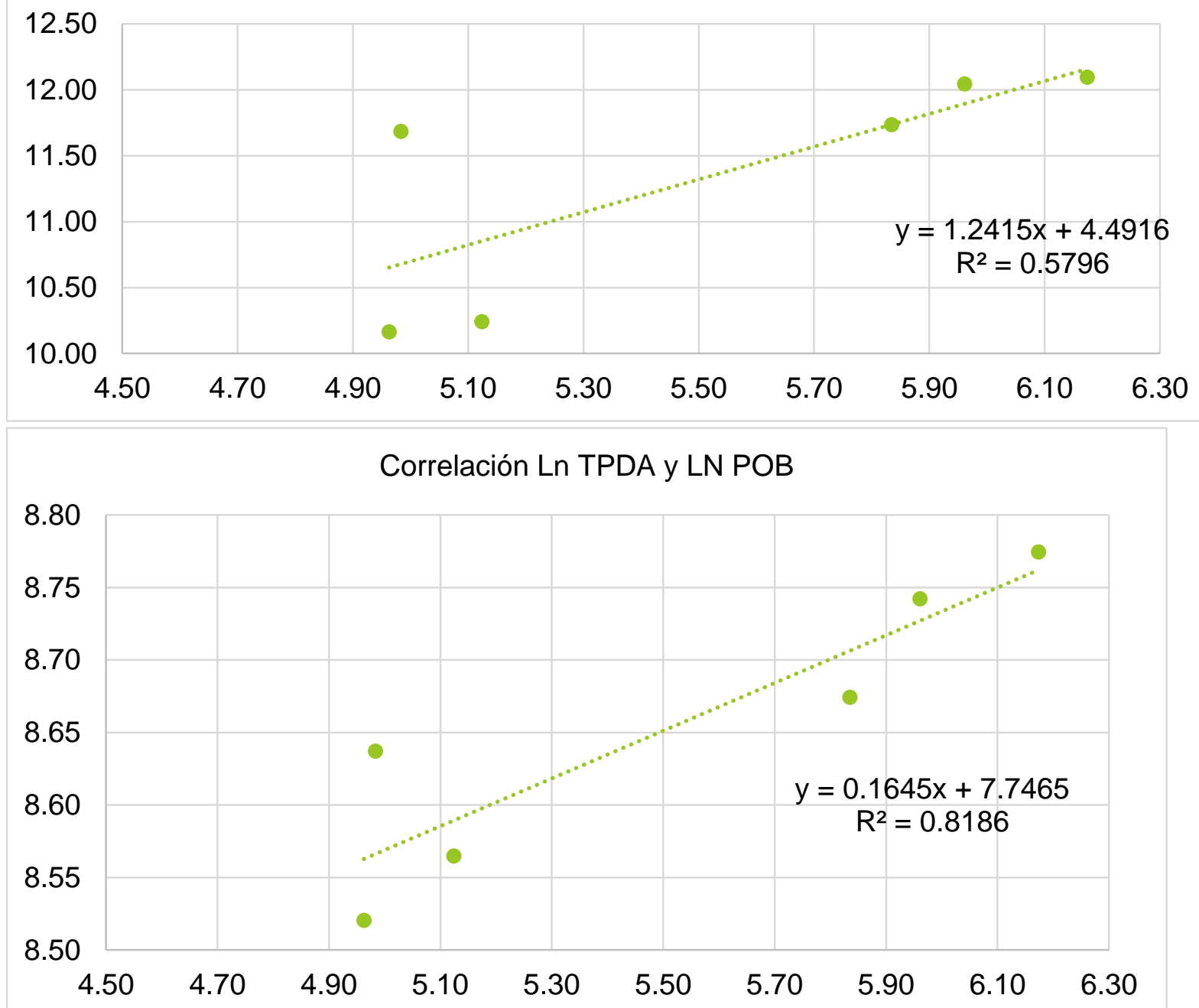

Figura 5. Correlaciones entre LN TPDA y datos macroeconómicos aplicando LN.

El Higo Revista Científica / Volumen 11. No. 01, pp. 43-68/ junio 2021 
Se debe de escoger los valores más lógicos de pendientes (elasticidades) y multiplicar por las tasas obtenidas. Estos resultados se presentan en la tabla 11.

Tabla 11. Tasas de crecimiento con correlaciones

\begin{tabular}{|c|c|c|c|}
\hline $\begin{array}{c}\text { Tasas } \\
\text { promedio }\end{array}$ & PIB $2.82 \%$ & $\begin{array}{l}\text { POB } \\
1.36 \%\end{array}$ & \\
\hline & Valor de pendiente & & \\
\hline $\begin{array}{l}\text { Correlación TPDA - } \\
\text { PIB }\end{array}$ & 388.59 & $\begin{array}{l}\text { Correlación TPDA - } \\
\text { Población }\end{array}$ & 3.6 \\
\hline $\begin{array}{l}\text { Correlación Ln TPDA } \\
\text { y Ln PIB }\end{array}$ & 1.2415 & $\begin{array}{l}\text { Correlación Ln TPDA } \\
\text { y LN POB }\end{array}$ & 0.1645 \\
\hline $\begin{array}{l}\text { Tasa de crecimiento } \\
=\text { Tasa promedio * } \\
\text { elasticidad }\end{array}$ & $2.82 * 1.2415=3.5 \%$ & & $1.36 * 3.6=4.9 \%$ \\
\hline
\end{tabular}

Si los valores son lógicos en rangos de variación establecidos, podrán ser usados las tasas. Por tanto, para este caso se designa:

Tasa de crecimiento general, igual a tasa de crecimiento vehículos livianos de $6.35 \%$, tasa de crecimiento para transporte de carga $3.5 \%$ y para transporte de pasajeros $4.9 \%$.

En nuevas carreteras, tal y como lo refiere Cal y Mayor (2018) debe ser considera el tráfico generado, que "consta de aquellos viajes vehiculares, distintos a los del transporte público, que no se realizarían si no se construye la nueva carretera (p. 220)" , el cual incorporará análisis y transformación de datos de producción a vehículos pudiendo estar en valores de $30 \%$ adicional al tráfico actual. Para este artículo no se hace referencia a la determinación de tráfico, dado que no es una nueva carretera, así como tampoco al cálculo de ESAL.

\section{CONCLUSIONES}

Para calcular el TPDA a partir de conteo de Tráfico Promedio Semanal se debe de multiplicar por factor día y factor temporada de los anuarios estadísticos de la estación de referencia seleccionada. Si el conteo es de solo tres días, recomendados el martes, miércoles y jueves, se deberá de multiplicar por factor día, factor semana y factor temporada.

De los anuarios estadísticos publicados por el MTI tomando en consideración los últimos cinco años, es necesario seleccionar los factores de expansión. Se debe seleccionar las estaciones de referencia cercanas a la estación de conteo. De no existir una estación referencial cercana, deberá hacer uso de estaciones de dependencia para para estimar el TPDA considerando el 
periodo de conteo. Para dar más sustento a las tasas de crecimiento, si los datos históricos lo permiten, podrá calcular los crecimientos promedios anuales, siendo un referente para la estimación.

Al emplear factores de expansión para estaciones de dependencia deberá de ser empleado el correspondiente al cuatrimestre que incluye el mes en el que este fue realizado. Para determinar la tasa de crecimiento a emplear, se debe analizar el comportamiento de la estación de corta duración o sumario de la cual se obtuvieron los factores de expansión para determinar el Tráfico Promedio Diario Anual.

Se debe de evaluar las tasas de crecimiento de tráfico acorde a tasa geométricas de crecimiento con al menos tres intervalos, tomando como referencia los crecimientos históricos consistentes de las estaciones de mayor cobertura siendo estos en rangos de 3-6\%. En este caso particular del periodo 2010 al 2018 para la Estación de Mayor Cobertura (ECM), San Marcos - Masatepe es de $6.78 \%$.

Es requerido que se continúen realizando esfuerzos para determinar las cargas reales actuantes sobres las estructuras de pavimentos, debiendo evaluar la distribución normalizada por ejes derivo a la sobrecarga y amplia variedad de la composición de flota vehicular en el país. Además, se deben establecer las estaciones de aforo o conteos manuales clasificados al menos $2 \mathrm{~km}$ alejados de zonas urbanas para evitar el efecto de repetición de ciclos de tránsito y efectos por movimientos de área urbana.

\section{REFERENCIAS}

Aragón, A., Hurtado, N., Torres, E. y Arévalo, A. (2012). Propuesta de los factores camión y espectros de carga para diseño de estructura de pavimento en las carreteras de Nicaragua. Universidad Nacional de Ingeniería. Recuperado de https://core.ac.uk/download/pdf/250141463.pdf

Ashhad, T., Cabrera, F. y Roa, O. (2021). Análisis del congestionamiento vehicular para el mejoramiento de vía principal en Guayaquil-Ecuador. Gaceta Técnica, 2, 1-14. Recuperado de https://www.redalyc.org/jatsRepo/5703/570363740001/html/index.html

Barraza Ortiz, E. E., Domínguez Solano, L. D. y Herrera Acosta, R. J. (2019). Cálculo del indice de capacidad de procesos usando media geométrica. Investigación e Innovación En Ingenierías, 7(2), 86-95. https://doi.org/10.17081/invinno.7.2.2851

BCN. (2021). Cuadros de Anuario de Estadísticas Macroeconómicas (1960 - 2019).

Berrones, L., Canos, P., Sánchez, D. y Martínez, J. (2018). Entregas tardías o incorrectas en el autotransporte de carga y su relación con las condiciones laborales de los choferes: Un modelo de regresión logística. Nova Scientia, 10(20), 413-441. 
https://doi.org/10.21640/ns.v10i20.1063

Cal y Mayor, R. C. J. (2018). Ingeniería de tránsito Fundamentos y aplicaciones (Novena edi). México: Alfaomega Grupo Editor.

Díaz, O. (2020). Contribución del transporte aéreo a la conectividad territorial. El caso de Colombia. EURE, 47(140). https://doi.org/10.7764/EURE.47.140.06

JICA. (2014). Informe del estudio preparatorio pala el proyecto de para la construcción del puente paso real en la república de Nicaragua. Managua. Recuperado de https://www.jica.go.jp/english/our_work/social_environmental/id/america/nicaragua/c8h0vm 000095q9e2-att/c8h0vm0000bzcsol.pdf

Kamplimath, H., Varuna, M., Kumar, V. y Bhargav, Y. (2013). Traffic growth rate estimation using transport demand elasticity method: a case study for national highway-63. International Journal of Research in Engineering and Technology, 02(13), 149-154. https://doi.org/10.15623/ijret.2013.0213026

López, C. y Pardo, S. (2019). El transporte de carga terrestre en el comercio internacional. Análisis comparativo entre Bogotá, Colombia y Santa Cruz de la Sierra, Bolivia. Ensayos de Economía, 29(54), 89-114. https://doi.org/10.15446/ede.v29n54.75022

Machado, R. (2017). Crecimiento económico e infraestructura de transportes y comunicaciones en el Perú. Economía, 40(79), 9-46. https://doi.org/10.18800/economia.201701.001

MTI. (2007). Revista de Conteo de Tráfico 2006. Managua.

MTI. (2016). Anuario de Aforo de Tráfico año 2015. Managua.

MTI. (2018). Anuario de Aforo de Tráfico año 2017. Managua.

MTI. (2019). Anuario de Aforo de Tráfico año 2018. Managua.

Normas Jurídicas de Nicaragua. Reglamento de ley general de transporte terrestre (2005). Nicaragua. Recuperado de https://n9.cl/2z7m

OCDE. (2018). Perspectivas económicas de América Latina 2018. (O. U. 2018, Ed.). Centro de Desarrollo de la Organización para la Cooperación y el Desarrollo Económicos - OECD. https://doi.org/10.1787/leo-2018-es

Rendón, J., Hernández, E. y Del Rio, H. (2019). Nueva generación de Modelos de transporte a través del uso de BIG DATA Caso San Salvador. Washington, D.C.: Transportation Research Board. https://doi.org/10.17226/25519 
Rodríguez, M., Mejía, M. y Zapata, S. (2015). La causalidad entre el crecimiento económico y la expansión del transporte aéreo: un análisis empírico para Chile. Revista de Economía Del Rosario, 18(1), 127-144. https://doi.org/10.12804/rev.econ.rosario.18.01.2015.04

SCT. (2016). Manual para obtener los Volúmenes de tránsito en carreteras. México: Secretaría de Comunicaciones y Transportes. Recuperado de https://www.sct.gob.mx/fileadmin/DireccionesGrales/DGST/Manuales/manual_volumen_de _transito/Manual_volumenes_2016_v2.pdf

Sun, L. (2016). LCCA-based design method for asphalt pavement. In Structural Behavior of Asphalt Pavements (pp. 549-600). Elsevier. https://doi.org/10.1016/B978-0-12-8499085.00008-0

Tennoy, A., Tonnesen, A. y Gundersen, F. (2019). Effects of urban road capacity expansion Experiences from two Norwegian cases. Transportation Research Part D: Transport and Environment, 69, 90-106. https://doi.org/10.1016/j.trd.2019.01.024

Zamora, A. y Pedraza, O. (2013). El transporte internacional como factor de competitividad en el comercio exterior. Journal of Economics Finance and Administrative Science, 18(35), 108118. https://doi.org/10.1016/S2077-1886(13)70035-0

\section{SEMBLANZA DE AUTOR}

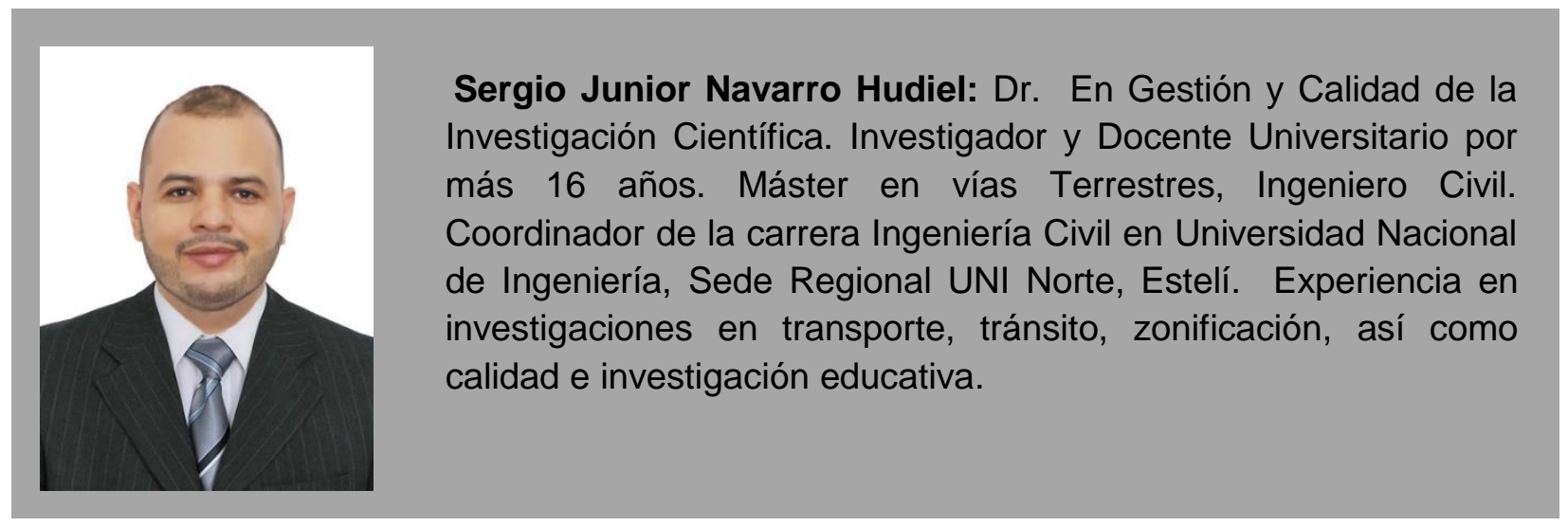

El Higo Revista Científica / Volumen 11. No. 01, pp. 43-68/ junio 2021 